\title{
Immature Reticulocyte Fraction Measurement
}

National Cancer Institute

\section{Source}

National Cancer Institute. Immature Reticulocyte Fraction Measurement. NCI Thesaurus. Code C102276.

The determination of the ratio of immature reticulocytes to total reticulocytes present in a sample. 